\title{
Nerve Stimulator Guided Lumbar Plexus and Parasacral Sciatic Nerve Block for above Knee Amputation in a High-Risk Patient: A Case Report
}

\author{
Penafrancia Catangui Cano, MD, DPBA, FPSA, EDRA* \\ Department of Anesthesiology, Philippine General Hospital, University of the Philippines Manila, Philippines
}

\begin{abstract}
Neuraxial block and general anesthesia are often the preferred anesthetic management for above knee amputation. In high-risk patients, however, with many co-morbidities, they may not be the ideal choice.

This is a case report of the factual benefits of nerve stimulator guided peripheral nerve block, a feasible option for reliable anesthesia for above knee amputation on a 64-year-old female, obese, diabetic, and septic patient with multiple comorbidities, who was scheduled for above knee amputation.

Ultrasound guidance is the most widely used nerve localization method of choice for peripheral nerve blocks. It can provide direct visualization of the needle, nerves, and the spread of the local anesthetic improving the success rate. In contrast, nerve stimulation relies on surface landmark identification, and physiological responses of neural structures to electrical stimulation which can be highly influenced by injectates and physiologic solutions affecting its efficacy. Since there was the absence of a working ultrasound machine when this emergency procedure was scheduled, the nerve stimulator guided technique was adopted in the performance of the peripheral nerve blocks.
\end{abstract}

\section{Introduction}

It is imperative that an adequate preoperative evaluation and a rational intraoperative and postoperative anesthetic management of patients with diabetes be exercised to avoid morbidity and mortality. Patients with longstanding diabetes mellitus often have long term complications, resulting in hypertension, cardiac ischemia, atherosclerosis, and renal failure among other syndromes [1].

Above knee amputation (AKA) is considered a high-risk surgery. Perioperative mortality rate ranges from $2.8 \%$ to $35 \%$ for AKA patients, which is even worse compared to below knee amputation (BKA) with a risk of $0.9 \%$ to $14.1 \%$ [25]. Lee, et al. identified six independent predictors of cardiac events for patients undergoing major noncardiac surgeries, which includes diabetes mellitus (DM), history of coronary artery disease, cerebrovascular disease, congestive heart failure (CHF), high-risk surgery, and serum creatinine level of $>2$ $\mathrm{mg} / \mathrm{dl}[6]$.

Considering that our patient exhibits all of the predictors enumerated neither neuraxial block nor general anesthesia would be the safest anesthetic management plan for the patient. Instead, an alternative technique in the form of peripheral nerve blocks which would provide cardiovascular stability [7] as well as prevent perioperative adverse event from occurring which would be a better choice, was performed.
This paper reports the use of peripheral nerve block for a very ill, diabetic patient who was scheduled for above knee amputation.

\section{Case Report}

A 64-year-old, $73 \mathrm{~kg}, 154.94 \mathrm{~cm}$, obese, female, patient was initially seen at the emergency department of the University of the Philippines-Philippine General Hospital because of neuroischemic foot ulcer of the $1^{\text {st }}$ and $2^{\text {nd }}$ toes of the left foot. The patient was referred and subsequently admitted to the Orthopedics department for further management.

While at the wards, referral to internal medicine service was carried out. She was diagnosed to have diabetes mellitus type 2 , uncontrolled, insulin requiring with retinopathy

*Corresponding author: Penafrancia Catangui Cano, MD, DPBA, FPSA, EDRA, Associate Professor, Head of the Section of Regional Anesthesia, Department of Anesthesiology, Philippine General Hospital, University of the Philippines Manila, Philippines

Accepted: July 23, 2020

Published online: July 25, 2020

Citation: Cano PC (2020) Nerve Stimulator Guided Lumbar Plexus and Parasacral Sciatic Nerve Block for above Knee Amputation in a High-Risk Patient: A Case Report. J Clin Anesth Pain Manag 4(1):107-113 
and neuropathy. She also had hypertension, chronic kidney disease, and congestive heart failure class II. History also revealed that she had a stroke in 2006 , which presented as left sided weakness from which she was able to recover with no residuals.

Further evaluation revealed sepsis with an elevated white blood cell count of $18.50 \times 10^{9} / \mathrm{L}$ although bleeding parameters were within normal limits. ECG findings showed an old anteroseptal wall infarct. On auscultation, crackles on both lung bases were observed together with pedal edema on the right foot.

Since there was no improvement on the wound healing of the neuroischemic foot despite the antibiotics, and with further worsening resulting in septicemia, the surgeons decided to schedule the patient for above knee amputation surgery. A referral to the anesthesiology department was then carried out.

Based from the pre-operative evaluation, considering the myriad of problems, a peripheral nerve block (PNB) in the form of combined lumbar plexus (LP) and parasacral sciatic nerveblock (PSNB) which would provide a unilateral lower extremity anesthesia, was decided as the anesthetic of choice to prevent any untoward perioperative cardiac morbidity. During the time of the anesthetic referral however, the ultrasound was not working and as such, the traditional technique of nerve stimulator and landmark guided LP and PSNB was planned.

On arrival at the operating room, a $0.9 \%$ Normal Saline Solution was flowing intravenously. Non-invasive monitors were attached (ECG, blood pressure cuff, pulse oximetry). Oxygen at $5 \mathrm{~L} / \mathrm{min}$ was administered via the Hudson mask. A central line was inserted at the right internal jugular vein to obtain a more reliable venous access and to be able to monitor the hemodynamic status of the patient. The initial blood pressure (BP) was 150/90. A dose of $1 \mathrm{mg}$ of Midazolam and 25 ug of Fentanyl was given intravenously to lessen the discomfort during positioning and during the conduct of the LP and PSNB, which are considered as deep type of PNBs [8]. The patient was then placed in the right lateral decubitus position. Using aseptic precautions, a left LP block was performed
(Figure 1). Four $\mathrm{cm}$ lateral to the spinous process of $L 4$ at the level of the iliac crest was the point of needle insertion [9]. The plexus was located using an insulated 21 gauge, $100 \mathrm{~mm}$ Vygon needle, which was attached to a nerve stimulator. It was advanced perpendicular to the skin until it contacted the L4 transverse process, after which it was slightly withdrawn and redirected cephalad and walked off. Contraction of the quadriceps muscle was elicited at a depth of $7 \mathrm{~cm}$ initially at a current of $1.5 \mathrm{~mA}$ until it was decreased to $0.5 \mathrm{~mA}$ with better localization of the nerve (Figure 2). The local anesthetic was slowly injected at increments of $5 \mathrm{ml}$, taking the time to aspirate and pause to investigate for any blood before delivery of the local anesthetic. Close monitoring of the heart rate and blood pressure was also exercised.

The PSNB block which was based from Mansour's technique [10] was subsequently performed. A line was drawn between the posterior superior iliac spine (PSIS) and ischial tuberosity. The same needle was inserted perpendicular to the skin $6 \mathrm{~cm}$ from the PSIS (Figure 3). Twitches of the hamstring, calf muscles, and plantar flexion of the foot were elicited at a depth of $7 \mathrm{~cm}$ with a current of $1.5 \mathrm{~mA}$ until it was lowered down to $0.5 \mathrm{~mA}$ with better positioning of the needle near the nerve. The volume of local anesthetic injected was $25 \mathrm{ml}$ each for the LP and PSNB for a total volume of $50 \mathrm{ml}$ which were composed of a mixture of Levobupivacaine (Chirocaine) $0.375 \%$ and saline with Adrenaline 1:400,000. Pinprick testing was used to assess sensation. Inability to move the left lower extremity completely transpired 20 mins after the PSNB, the second block, was performed.

With the persistence of the same blood pressure taken upon arrival at the operating room, $5 \mathrm{mg}$ of Nitroglycerin patch was placed on the left anterior chest wall before surgery commenced. The BP was maintained between 120$130 / 80 \mathrm{mmHg}$ from the start of surgery until it was finished while the CVP pressure remained constant between 9-11 $\mathrm{mmHg}$. The patient was comfortable and did not complain of any pain during the whole duration of the procedure which lasted for 2 hours and 30 minutes (Figure 4).

\section{Discussion}

Amputation of the lower extremity is a cause of ma-

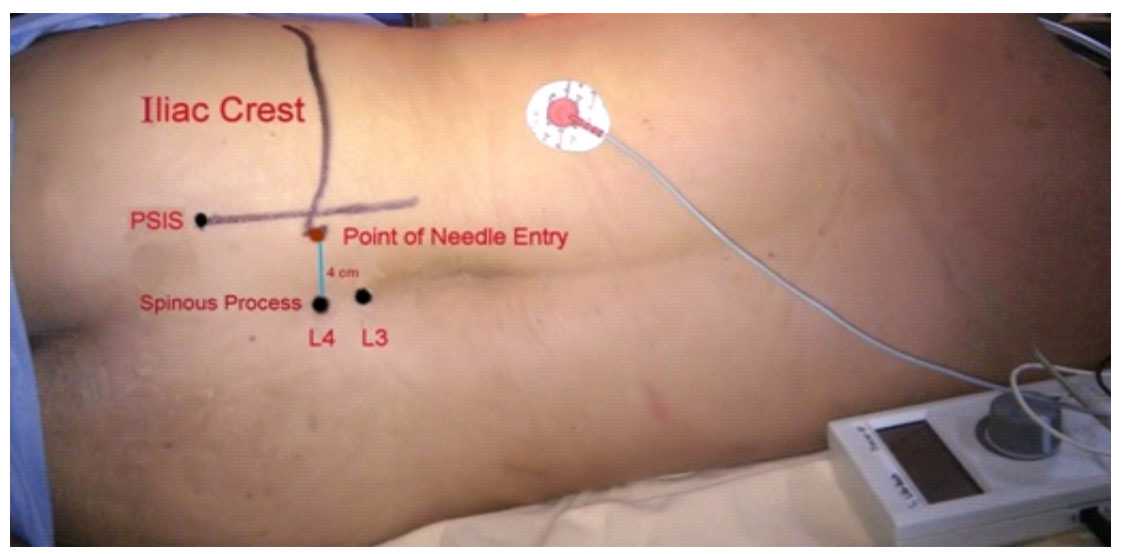

Figure 1: Lumbar plexus block. 


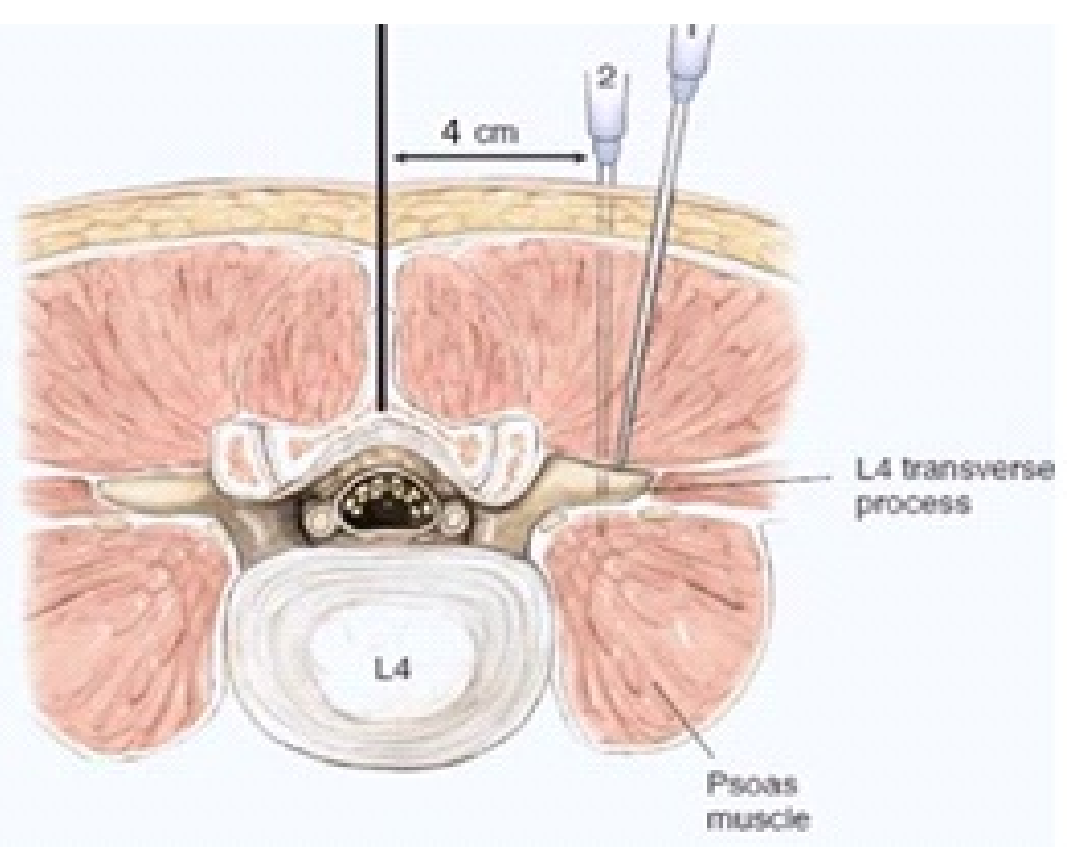

Figure 2: Lumbar plexus block (axial view). Line is drawn $4 \mathrm{~cm}$ from the $L 4$ spinous process. The needle is inserted perpendicularly to the skin after which it is redirected cephalad after contacting the transverse process.

Copyright 2009, Anita Impagliazzo [20].

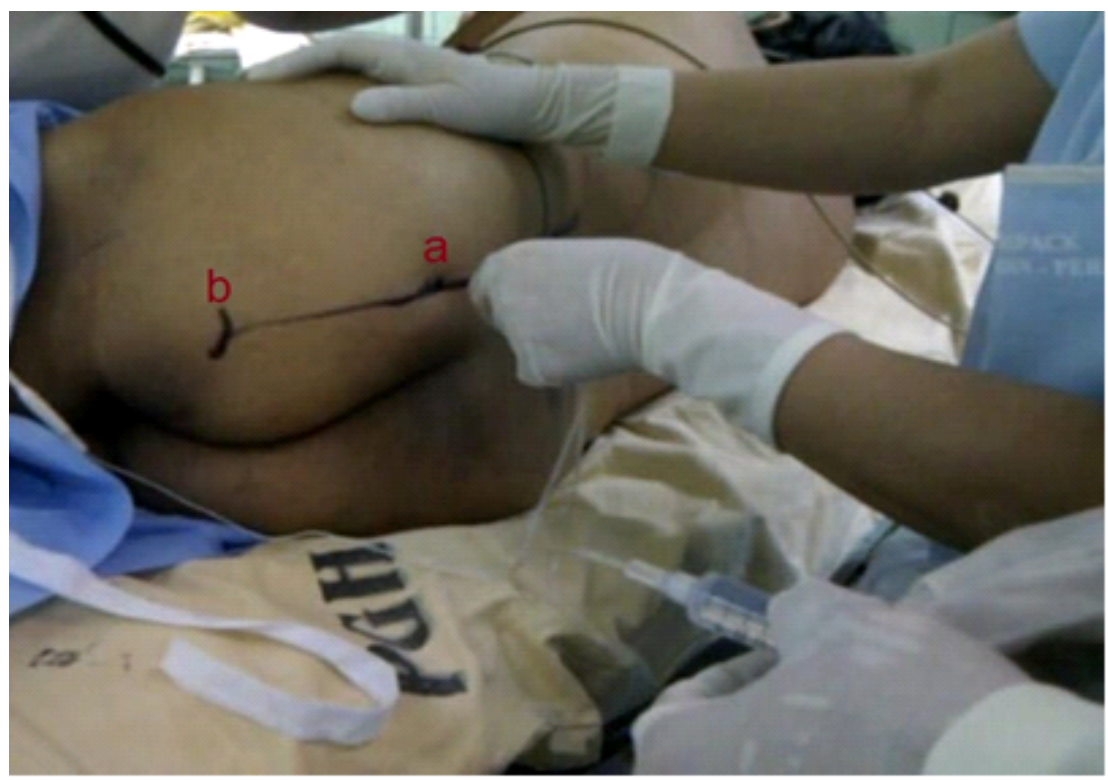

Figure 3: Parasacral nerve block of Mansour. The injection site is $6 \mathrm{~cm}$ from the posterior superior iliac spine. (a) Injection site; (b) Ischial tuberosity.

jor concern because of its propensity to result in significant perioperative morbidity and mortality. This is often attributed to the presence of associated medical problems namely diabetes mellitus, chronic kidney disease, cardiac co-morbidities (congestive heart failure, arrhythmia, myocardial infarction), hypertension, pneumonia, sepsis, and cerebrovascular disease $[3,6]$. A retrospective review of 959 major amputations reported a cardiac complication rate of 10.2 percent. Complications included heart failure (4.2 percent), arrhythmias (2.6 percent), and myocardial infarction (3.4 percent) [3]. Opera- tive mortality rate is even higher in AKA patients as compared to BKA patients [2-5]. Therefore, in order to limit the complications and to prevent untoward outcomes, the constitution of preventive strategies is important to reduce the morbidity and mortality associated with the surgical procedure.

Regional anesthesia (RA) in the form of neuraxial blocks, namely spinal and epidural anesthesia, are too often the preferred choice for AKA. Based on the meta-analysis done by Rodgers, et al., which included 141 trials covering 9559 pa- 


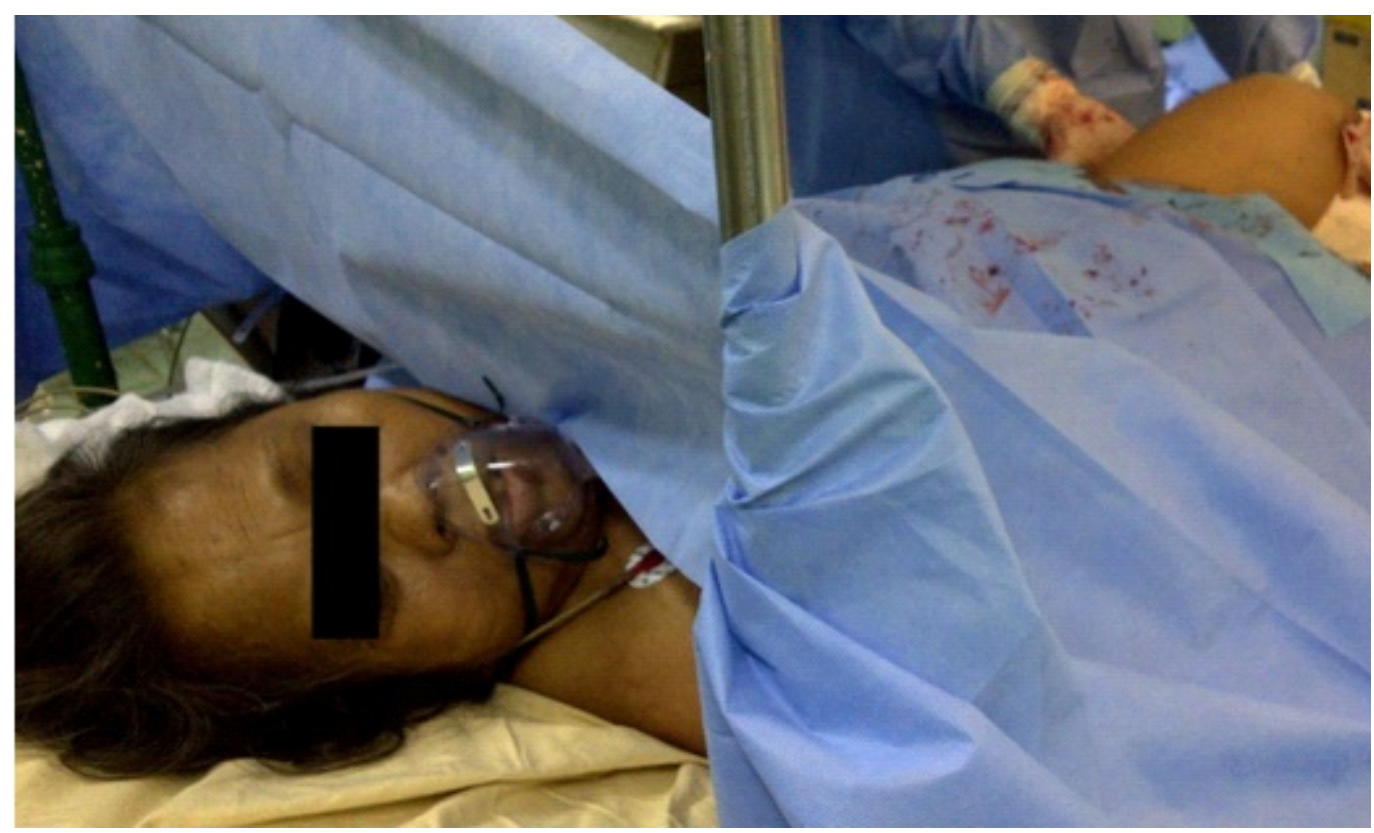

Figure 4: Patient after the above knee amputation.

tients, neuraxial block reduces peri-operative mortality by about one third [11]. Compared to general anesthesia (GA), regional anesthesia results in a lower incidence of deep vein thrombosis, pulmonary embolism, blood loss, pneumonia, respiratory depression, myocardial infarction, and renal failure $[11,12]$. RA allows the patient to be awake during the procedure reducing the incidence of postoperative confusion [13]. Furthermore, it can suppress the stress response to surgery during periods of intense stimulation, such as during intubation and extubation, avoiding fluctuations in blood pressure preventing perioperative complications [14].

Despite the advantages of neuraxial block, this technique can cause profound hypotension secondary to sympathetic blockade and autonomic neuropathy in a diabetic patient, which may further aggravate the co-existing coronary, cerebrovascular, and renal problems [15]. Considering all the mentioned complications, PNB, which is another form of RA, was the preferred type of anesthetic that was conducted for the patient. PNB is a useful, safe, and effective technique for lower limb amputation in older and diabetic patients with poor general conditions who are classified as high-risk $[16,17]$. These blocks result in a lower incidence of intra-operative hypotension and post-operative admission to the ICU as compared to GA or neuraxial block $[16,18,19]$. Inaddition, PNB minimizes blood loss, decreases post-operative opioid requirements, inhibits nausea and vomiting, prohibits urinary retention, prevents spinal headache, and offers excellent post-operative analgesia $[20,21]$. However, since the performance of a successful PNB requires training, experience and adequate skill, it remains under-utilized [22].

For this case, the PNBs that have been utilized were lumbar plexus and parasacral sciatic nerve block which are the recommended blocks for such types of surgeries [22-24]. A clear understanding of the anatomy of the nerve innervation of the lower limb and the technical aspects in the perfor- mance of PNB is of utmost importance to attain success. The nerve supply of the lower limb is derived from the lumbar plexus (LP) and the sacral plexus (SP). In order to provide a satisfactory unilateral anesthesia in the lower extremity, both of these two plexuses should be blocked.

The LP includes the ventral divisions from L1-4. The main nerves that have to be considered when performing a LP block are the femoral, lateral femoral cutaneous and the obturator nerves, which are derived from the roots of L2-L4. They are the nerves that provide muscular and cutaneous branches to the anterior, lateral, and medial portion of the thigh [9,21]. All of these nerves are embedded in an anatomical space in the psoas major muscle called the psoas compartment [25]. Thus, the most consistent approach for the entire LP to be blocked with a single injection is through the posterior approach LP block, which is also known as the Psoas compartment block [26]. Karmakar described a technique of ultrasound-guided LP block that was successfully used in conjunction with PSNB for anesthesia in a series of patients undergoing emergency lower limb surgery [27]. A few years later, Sauter introduced the Shamrock technique as a new LP approach where ultrasound scanning which is performed at the patient's flank in a transverse plane gives an improved view of the lumbar plexus and the surrounding anatomy [28].

On the other hand, the sacral plexus are derived from the L4 through S3 nerve roots. The branches that are relevant for anesthesia of the lower extremity are the sciatic nerve and the posterior femoral cutaneous nerve of the thigh. These nerves provide motor and sensory innervations to the posterior thigh respectively. Compared with other more distal approaches to the sciatic nerve, the PSNB technique consistently anesthetizes both of the two branches $[26,29]$. This block provides excellent surgical anesthesia and is coupled with a high success rate [30-32]. Thus, LP block combined with PSNB provides complete anesthesia of the ipsilateral lower limb which 
is effective for above knee amputation $[7,17,21,26,27,33,34]$.

At present, ultrasound guidance is the most widely used nerve localization technique of choice for PNB [35]. Its popularity is attributed to its ability to provide direct visualization of the needle, the nerves, and the spread of the local anesthetic [36-38]. The use of ultrasound in lower extremity blocks had shown to decrease the block performance time, shorten the block onset, prevent inadvertent vascular puncture and multiple needle passes and it can improve the success rate [26,36-38]. Moreover, since there is improved accuracy in the placement of the local anesthetic relative to the nerve structures, lower volumes are needed to achieve a successful block which would help in preventing local anesthetic systemic toxicity (LAST) [36,38].

For our patient, the landmark based approach with the guidance of the nerve stimulator was conducted since the ultrasound machine at the hospital during the time this block was carried out was not working. In the past, nerve stimulation was considered as the standard tool for anesthesiologists to locate the nerve structures for peripheral nerve blocks. This instrument which was introduced by Perthes in 1912 [39] provided an objective means for identifying needle-nerve proximity which was an integral step towards transforming regional anesthesia into "science" [40].

In comparison to the ultrasound guided technique, neurostimulation relies on anatomical surface landmark identification and physiological responses of neural structure to electrical stimulation which can be highly influenced by injectates and physiologic solutions limiting its functions and reducing its level of efficacy [40]. Although ultrasound has become the main tool in the performance of RA techniques, anesthesiologists nevertheless must be adequately trained in nerve stimulator guided RA [41].

Complications secondary to LP block have been reported, which include total spinal anesthesia [42], epidural spread of the local anesthetic [43], retroperitoneal hematoma [44], renal subcapsular hematoma [45], and inadvertent intravascular injection of cardiotoxic local anaesthetics [46]. These issues, combined with unfamiliarity with the technique, could be reasons for reluctance in the use of the LP block leading to its underutilization. These complications however can be prevented with adequate knowledge of anatomy, avoidance of high injection pressure, slow fractionated injection of local anesthetic and frequent aspiration prior to injection to ensure that no blood is drawn during the performance of the block while epinephrine should be incorporated as an intravascular marker $[26,39,47]$. Most importantly, vigilance should always be maintained. On the other hand, PSNB may cause paresthesia, pain at the injection site as well as LAST [48].

Despite its usefulness, combined LP and PSNB have several disadvantages. Compared to neuraxial block, it is also technically more difficult to perform. Furthermore, a larger volume of local anesthetic is required to anesthetize the entire lower extremity predisposing patients to a greater risk of local anesthetic toxicity. The study of Vanterpool showed that large doses of local anesthetic can be used safely for LP block either alone or when combined with sciatic nerve block for the maximum plasma concentration they obtained is below the threshold for toxicity [49]. They also recommended that the dose should be adjusted in patients with co-morbidities. In the same manner, Simon, et al. demonstrated that high doses of Mepivacaine for combined LP and sciatic nerve block displayed a low plasma concentration but they suggested that the addition of epinephrine to the local anesthetic solution considerably lowered the plasma concentration [50]. Since a rapid rise in plasma concentration after injection appears to occur when the two blocks were combined [49], a slow, gentle injection must be practiced since a forceful, rapid injection of the local anesthetic may carry a much higher probably of LAST [2].

With the numerous co-morbidities of our patient, Levobupivacaine (Chirocaine), the pure $\mathrm{S}$ enantiomer of racemic Bupivacaine which is less cardiotoxic and neurotoxic, was the local anesthetic that was injected to the patient for both blocks $[51,52]$. To further prevent local anesthetic toxicity, the concentration of Levobupivacaine was decreased to $0.375 \%$ which is still adequate for surgical anesthesia [33].

\section{Conclusion}

In summary, combined lumbar plexus and parasacral sciatic nerve block is an effective technique that can be utilized when confronted with a high-risk patient for above knee amputation. This case report also shows that nerve stimulator guided peripheral nerve blocks can be safely and successfully performed for such type of a procedure. Despite the significant benefits of ultrasound over nerve stimulation, trainees must not abandon and should remain experts in neurostimulation.

In situations where the performance of peripheral nerve blocks is vital to the life of the patient and the ultrasound machine is not available, the well rounded regional anesthesiologists will still be able to execute with confidence the desired nerve blocks with the use of the nerve stimulator.

\section{References}

1. Gautam A, Baluch A, Kaye A, et al. (2009) Modern strategies for the anesthetic management of the patient with diabetes. Middle East J Anaesthesiol 20: 187-197.

2. Subramaniam B, Pomposelli F, Talmor D, et al. (2005) Perioperative and long term morbidity and mortality after above-knee and below-knee amputations in diabetics and nondiabetics. Anesth Analg 100: 1241-1247.

3. Aulivola B, Hile D, Hamdan A, et al. (2004) Major lower extremity amputation. Outcome of a modern series. Arch Surg 139: 395399.

4. Bunt T, Manship L, Bynoe R, et al. (1984) Lower extremity amputation for peripheral vascular disease: A low risk operation. Am Surg 50: 581-584.

5. Kazmers A, Perkins A, Jacobs L (2000) Major lower extremity amputation in veterans affairs medical centers. Ann Vasc Surg 14: 216-222.

6. Lee T, Marcantonio E, Mangione C, et al. (1999) Derivation and prospective validation of a simple index for prediction of cardiac risk of major noncardiac surgery. Circulation 100: 1043-1049. 
7. Baddoo $\mathrm{H}$ (2009) A preliminary report on the use of peripheral nerve blocks for lower limb amputations. Ghana Med J 43: 2428.

8. Deschner B, Robards C, Daquan X, et al. (2009) A comprehensive review of lower extremity peripheral nerve blocks. NYSORA 12 $11-22$

9. Hadzic A (2007) Textbook of regional anesthesia and acute pain management.

10. Mansour NY (1993) Reevaluating the sciatic nerve block: Another landmark for consideration. Reg Anesth 18: 322-323.

11. Rodgers A, Walker N, Schug S, et al. (2000) Reduction of postoperative mortality and morbidity with epidural or spinal anesthesia: Results from overview of randomised trials. BMJ 321: 1493-1497.

12. Mauermann W, Shilling A, Zuo Z, et al. (2006) A comparison of neuraxial block versus general anesthesia for elective total hip replacement: A meta-analysis. Anesth Analg 103: 1018-1025.

13. Mohanty S, Rosenthal R, Russell M, et al. (2016) Optimal perioperative management of the geriatric patient: $A$ best practices guideline from the American college of surgeons NSQIP and the American geriatrics society. J Am Coll Surg 222: 930-947.

14. Robertshaw H, Hall G (2006) Diabetes mellitus: Anaesthetic management. Anaesthesia 61: 1187-1190.

15. McAnulty G, Robertshaw H, Hall G (2000) Anaesthetic management of patients with diabetes mellitus. BJA 85: 80-90.

16. Kumar R, Singh A, Talib H (2001) Amputation of lower extremity in diabetic and high-risk patients under peripheral nerve blocks (combined sciatic and 3-in-1 femoral blocks). Kuwaiti Medical Journal 33: 310-316.

17. Tharwat A (2011) Combined posterior lumbar plexus-sciatic nerve block versus combined femoral-obturator-sciatic never block for ACL reconstruction. Local Reg Anesth 4: 1-6.

18. Najar Z, Hassan M, Khalib H, et al. (2000) Combined sciatic-paravertebral nerve block vs. general anesthesia for fractured hip of the elderly. Middle East J Anaesthesiol 15: 559-568.

19. Eyrolle L, Zetlaoui P, Belbachir A, et al. (1998) Regional anesthesia for femoral neck fracture surgery: Comparison of lumbar plexus block and spinal anesthesia. Br JAnaesth 80: 383.

20. Dilger J (2000) Lower extremity nerve blocks. Anesthesiol Clin North Am 18: 319-340.

21. Hogan M, Grant R, Lee L (2009) Analgesia for total hip and knee arthroplasty: A review of lumbar plexus, femoral, and sciatic nerve blocks. Am J Orthop (Belle Mead NJ) 38: E129-E133.

22. Bendtsen TF, Haskins S, Kølsen Petersen JA, et al. (2016) Doultrasound-guided regional blocks signify a new paradigmin high-risk patients? Best Pract Res Clin Anaesthesiol 30: 191-200.

23. Enneking FK, Chan V, Greger J, et al. (2005) Lower-extremity peripheral nerve blockade: Essentials of our current understanding. Reg Anesth Pain Med 30: 4-35.

24. Gadsden J (2012) Indications for peripheral nerve blocks. In Hadzic A, Hadzic's Peripheral nerve blocks and anatomy for ultrasound-guided regional anesthesia. (edn), The McGraw-Hill Companies, New York, 81-94.

25. Murray J, Derbyshare S, MO Shields (2010) Lower limb blocks. Anaesthesia 65: S57-S66.

26. Iwata T, Lakshman S, Hadzic A, et al. (2005) Peripheral nerve blocks for perioperative management of patients having orthopedic surgery or trauma of the lower extremity. Bosn J Basic Med Sci 5: 5-19.

27. Karmakar M, Ho A, Li X, et al. (2008) Ultrasound-guided lumbar plexus block through the acoustic window of the lumbar ultrasound trident. Br J Anaesth 100: 533-537.

28. Sauter AR (2013) The "shamrock method" a new and promising technique for ultrasound guided lumbar plexus blocks. BJA 111.

29. Morphett S (2009) Nerve blocks for anaesthesia and analgesia of the lower limb-A practical guide: Femoral, lumbar plexus, sciatic. Update in Anesthesia 10: 56-65.

30. Morris G, Lang S, Dust W, et al. (1997) The parasacral sciatic nerve block. Reg Anesth 22: 223-238.

31. Cuvillon P, Ripart J, Jeannes P, et al. (2003) Comparison of the parasacral approach and the posterior approach, with single-and double-injection techniques to block the sciatic nerve. Anesthesiology 98: 1436-1441.

32. Ripart J, Cuvillon P, Nouvellon E, et al. (2005) Parasacral approach to block the sciatic nerve: A 400-case survey. Reg Anesth Pain Med 30: 193-197.

33. Kocum A, Turkoz A, Ulger $\mathrm{H}$, et al. (2007) Ropivacaine $0.25 \%$ is as effective as bupivacaine $0.25 \%$ in providing surgical anaesthesia for lumbar plexus and sciatic nerve block in high-risk patients: Preliminary report. Anaesth Intensive Care 35: 510-514.

34. de Visme V, Picart F, Le Jouan R, et al. (2000) Combined lumbar and sacral plexus block compared with plain bupivacaine spinal anesthesia for hip fractures in the elderly. Reg Anesth Pain Med 25: 158-162.

35. Clendenen S, Robards C (2010) The role of ultrasound and regional anesthesia. International Anesthesiology Clinics 48: 13-20.

36. Marhofer P, Harrop-Griffiths W, Kettner S, et al. (2010) Fifteen years of ultrasound guidance in regional anaesthesia: Part $1 . \mathrm{Br} \mathrm{J}$ Anaesth 104: 538-546.

37. Griffin J, Nicholls B (2010) Ultrasound in regional anesthesia. Anaesthesia 65: S1-S12.

38. Abrahams M, Aziz M, Fu R, et al. (2009) Ultrasound guidance compared with electrical neurostimulation for peripheral nerve block: A systematic review and meta-analysis of randomized controlled trials. Br J Anaesth 102: 408-417.

39. Castellvi J (2012) Is neurostimulation in regional anesthesia still worth knowing? Techniques in Regional Anesthesia and Pain Management 16: 136-139.

40. Tsui, B (2007) Ultrasound-guidance and nerve stimulation: Implications for the future practice of regional anesthesia. Can J Anesth 54: 165-170.

41. Tornero C, Font $L$ (2012) Training on regional anesthesia-from neurostimulation to ultrasound. Techniques in Regional Anesthesia and Pain Management 3: 131-135.

42. Pousman R, Mansoor Z, Sciard D (2003) Total spinal anesthetic after continuous posterior lumbar plexus block. Anesthesiology 98: 1281-1282.

43. Mannion S (2004) Epidural spread depends on the approach used for posterior lumbar plexus block. Can J Anaesth 51: 516517.

44. Weller R, Gerancher J, Crews J, et al. (2003) Extensive retroperitoneal hematoma without neurologic deficit in two patients who 
underwent lumbar plexus block and were later anticoagulated. Anesthesiology 98: 581-585.

45. Aida S, Takahashi H, Shimoji K (1996) Renal subcapsular hematoma after lumbar plexus block. Anesthesiology 84: 452-455.

46. Pham-Dang C, Beaumont S, Floch H, et al. (2000) Acute toxic accident following lumbar plexus block with bupivacaine. Ann $\mathrm{Fr}$ Anesth Reanim 19: 356-359.

47. de Leeuw MA, Zuurmond WWA, Perez RSGM (2011) The psoas compartment block for hip surgery: The past, present, and future. Anesthesiol Res Pract 2011: 159541.

48. Petitjeans F, Mion G, Puidupin M, et al. (2002) Tachycardia and convulsions induced by accidental intravascular ropivacaine injection during sciatic block. Acta Anaesthesiol Scand 46: 616-617.
49. Venterpool S, Steele S, Nielsen K, et al. (2006) Combined lumbar-plexus and sciatic-nerve blocks: An analysis of plasma ropivacaine concentrations. Reg Anesth Pain Med 31: 417-421.

50. MA Simon, MJ Gielen, AJ Lagerwerf, et al. (1990) Plasma concentrations after high doses of mepivacaine with epinephrine in the combined psoas compartment/sciatic nerve block. Reg Anesth 15: $256-260$.

51. Bardsley H, Gristwood R, Baker H, et al. (1998) A comparison of the cardiovascular effects of Levobupivacaine and rac-bupivacaine following intravenous administration to healthy volunteers. Br J Clin Pharmacol 46: 245-249.

52. Arias M (2002) Levobupivacaine: A new safer long acting local anesthetic with less cardiac and neurotoxicity. Update in Anesthesia 14: 23-25. 